\title{
A New Hybrid Nelder-Mead Particle Swarm Optimization for Coordination Optimization of Directional Overcurrent Relays
}

\author{
An Liu ${ }^{1,2}$ and Ming-Ta Yang ${ }^{3}$ \\ ${ }^{1}$ Department of Computer Science and Information Engineering, St. John's University, No. 499, \\ Section 4, Tam King Road, Tamsui District, New Taipei City 25135, Taiwan \\ ${ }^{2}$ Graduate Institute of Computer and Communication Engineering, National Taipei University of \\ Technology, No. 1, Section 3, Chung Hsiao East Road, Taipei 10608, Taiwan \\ ${ }^{3}$ Department of Electrical Engineering, St. John's University, No. 499, Section 4, Tam King Road, \\ Tamsui District, New Taipei City 25135, Taiwan
}

Correspondence should be addressed to Ming-Ta Yang, mtyang@mail.sju.edu.tw

Received 18 February 2012; Revised 26 April 2012; Accepted 10 May 2012

Academic Editor: Jianming Shi

Copyright (C) 2012 A. Liu and M.-T. Yang. This is an open access article distributed under the Creative Commons Attribution License, which permits unrestricted use, distribution, and reproduction in any medium, provided the original work is properly cited.

\begin{abstract}
Coordination optimization of directional overcurrent relays (DOCRs) is an important part of an efficient distribution system. This optimization problem involves obtaining the time dial setting (TDS) and pickup current $\left(I_{p}\right)$ values of each DOCR. The optimal results should have the shortest primary relay operating time for all fault lines. Recently, the particle swarm optimization (PSO) algorithm has been considered an effective tool for linear/nonlinear optimization problems with application in the protection and coordination of power systems. With a limited runtime period, the conventional PSO considers the optimal solution as the final solution, and an early convergence of PSO results in decreased overall performance and an increase in the risk of mistaking local optima for global optima. Therefore, this study proposes a new hybrid Nelder-Mead simplex search method and particle swarm optimization (proposed NM-PSO) algorithm to solve the DOCR coordination optimization problem. PSO is the main optimizer, and the Nelder-Mead simplex search method is used to improve the efficiency of PSO due to its potential for rapid convergence. To validate the proposal, this study compared the performance of the proposed algorithm with that of PSO and original NM-PSO. The findings demonstrate the outstanding performance of the proposed NM-PSO in terms of computation speed, rate of convergence, and feasibility.
\end{abstract}

\section{Introduction}

Transmission lines are exposed to the environment and stretch long distances, which increases the probability of failure far beyond that of other components of the network, including generators, transformers, and switchgear equipment. 
A transmission network is usually divided according to function: (1) transmission lines between various major substations forming the backbone of the network; (2) subtransmission lines connecting substations to load centers or major users; (3) distribution lines between load centers and end users.

Lines from substations or load centers are often distributed in the form of radial feeders. Radial feeders only require the installation of relays and breakers at the ends of the various lines from which the power is sent. When a fault occurs in most radial feeders, the fault current will be greater than the load current with no reverse fault current. As a result, these types of radial feeders can be protected using nondirectional overcurrent relays.

If the protected line is installed with power supplies at both ends, such as in the case of loop networks, the fault current may be fed from the left or right in the event of reverse external failure. In this case, relay malfunctions may occur only if nondirectional overcurrent relays are used for protection, as these relays cannot be coordinated. Directional overcurrent relay (DOCR) is a method to improve protection. DOCR is designed to function only in the event of a unidirectional fault current.

The study of coordination problems in electrical power systems has become increasingly important in recent years. Economic considerations have propelled DOCR into widespread use as the primary protection for distribution systems and as the backup protection for transmission systems. When working with a DOCR system, operators must set time dial setting (TDS) and pick up current $\left(I_{p}\right)$ values according to the coordination relationship of the primary/backup (P/B) pairs to fully secure protection for the entire system.

In recent years, several optimization techniques have been proposed for the optimal coordination of DOCRs. Urdaneta et al. applied a minima optimization approach to determine the TDS values for preset $I_{p}$ values for fixed and multiple power system network configurations [1]. Abyaneh et al. obtained optimum coordination by considering linear and nonlinear models of relay characteristics and changes in network configuration [2]. Birla et al. demonstrated the simultaneous optimization of all DOCR settings with nonlinear relay characteristics using a sequential quadratic programming method [3]. In [4], a genetic algorithm was selected as the tool to solve the DOCR coordination problem, which included nonlinear constraints. The results of [5] reveal that the advantage of the proposed interval method for the DOCR coordination problem provides robust support against uncertainty in the topology of the network. Bedekar and Bhide used a genetic algorithm and nonlinear programming method to systematically determine initial and final values of the time multiplier and plug settings for optimal DOCR coordination [6].

In general, PSO algorithms are not easily trapped in local optima; however, the convergence rate is slow, and optimization problems with constraints cannot be effectively solved. Zeineldin et al. proposed an approach using a modified particle swarm optimization (PSO) algorithm to calculate the optimal relay settings, formulating the coordination problem as a mixed-integer nonlinear programming problem [7]. In [8], the problem of setting the DOCR was formulated and solved as a linear programming problem; a modified PSO was also applied. The major goal of this study was to investigate the feasibility of applying a Nelder-Mead simplex search method and a particle swarm optimization (NMPSO) methodology to address the coordination optimization of a DOCR distribution system.

We have divided the remainder of this paper into three sections. The first provides an introduction to the theoretical foundations of the research, involving the modeling of DOCR coordination problems. The proposed optimization algorithm includes a constraint handling method, an NM simplex search method, a PSO algorithm, and an NM-PSO method. We utilized IEEE 8- and 14-bus test systems to verify the feasibility of the proposed algorithm. 
The results show that the proposed method, comprising a linear programming (LP) problem and a mixed-integer nonlinear programming (MINLP) problem, is capable of overcoming the relay coordination problem of a power system. The combined approach effectively increases the convergence rate of calculation and enhances the capability of the PSO when processing under constraints. Finally, we discuss the results and draw conclusions.

\section{DOCR Coordination Problem}

The main purpose of the DOCR coordination problem is to determine the TDS and $I_{p}$ values of each relay in a power system. The optimal operating times of the primary relays are then minimized, and coordination pairs of the $\mathrm{P} / \mathrm{B}$ relays and coordination constraints are obtained. The DOCR coordination optimization problem in a power system can be described as follows:

$$
\min J=\sum_{i=1}^{n} w_{i} t_{i k}
$$

where $n$ is the number of relays in zone $k$ of a power network, and $w_{i}$ is a coefficient indicating the probability of a fault occurring on the $i$ th line in zone $k$ of a power network. In general, the value of $w_{i}$ is either 1 or 0 . The variable $t_{i k}$ indicates the operating time of relay $i$ for a close-in fault in zone $k$.

The coordination constraints between the primary relay $i$ and the backup relay(s) $j$ are as follows:

$$
t_{j k}-t_{i k} \geq \mathrm{CTI}
$$

where $t_{j k}$ reveals the operating time of relay $j$, and the relay is the backup relay of relay $i$. CTI is the minimum coordination time interval; its value ranges from 0.2 to $0.5 \mathrm{~s}$. In this study, a CTI of $0.2 \mathrm{~s}$ was chosen.

The function for the nonlinear relay characteristics is based on IEEE standard C37.1121996 and is represented as follows:

$$
t_{i}=\mathrm{TDS}_{i} \times\left(\frac{28.2}{\left(I_{\mathrm{fi}} / I_{\mathrm{pi}}\right)^{2}-1}+0.1217\right)
$$

where $\mathrm{TDS}_{i}$ and $I_{\mathrm{pi}}$ are the time dial setting and the pickup current setting of the $i$ th relay, respectively. $I_{\mathrm{fi}}$ is the short-circuit fault current passing through the $i$ th relay.

The constants and exponent in (2.3) define the shape of the extremely inverse trip characteristics.

The results of this research not only describe the methodology of DOCR coordination optimization but also demonstrate the feasibility of the TDS and $I_{p}$ settings of the relays. In general, DOCR allows for a continuous TDS value, but a discrete $I_{p}$ setting. To satisfy this requirement, this study explored both linear and nonlinear programming for DOCR coordination optimization. The variable TDS is optimized according to a predefined $I_{p}$ for each $\mathrm{DOCR}$, and this optimization problem can be viewed as a linear programming (LP) problem. 
For the nonlinear programming (NLP) problem, variables TDS and $I_{p}$ are optimized for each DOCR. In the LP or NLP problem of the DOCR coordination optimization, the TDS values can range continuously from 0.1000 to 1.1000 , and the $I_{p}$ values can range discretely between 10 and 1000 with a step size of 1, depending on the close-in fault current for each relay.

\section{Proposed Optimization Algorithm}

PSO is a random optimization technology developed by Eberhart and Kennedy in 1995 [9], who were inspired by simulating the intelligence of swarming bird flocks. PSO shares many similarities with evolutionary computation techniques such as genetic algorithms (GAs) [6]. The problem is initialized with a population of feasible random solutions; however, PSO contains no genetic operations, such as crossover or mutation.

Another important feature of PSO is that each particle has memory. PSO's information sharing mechanism differs greatly from that of GAs. In GAs, chromosomes mutually share information, and therefore, the movement of the population as it approaches the best area is relatively even. In PSO, the possible individual elements of PSO algorithms are called particles. The global best particle gives information to other particles and updates the movement direction and speed of each particle.

Based on the PSO method, we propose the NM-PSO method for solving the constrained optimization problem. The following section introduces the basic principles of NMPSO, including constraint-handling methods, Nelder-Mead (NM) simplex search, and PSO.

\subsection{Constraint-Handling Methods}

Constraint handling is a major concern when applying PSO algorithms to solve constrained optimization problems. This is because the traditional search operators of PSO algorithms are blind to constraints. Thus far, the most commonly used constraint handling methods for PSO are the penalty and repair methods.

The gradient-based repair method was addressed by [10,11]. This method adopts gradient information derived from the constraint set to gradually repair an infeasible solution by directing the infeasible solution toward a feasible area. Because the constraints of the DOCR coordination optimization are not complicated, this method is highly suitable. Furthermore, because DOCR coordination optimization has no equality constraints, equality constraint equations can be ignored. This method is described below.

(1) For a random solution, determine the degree of constraint violation $\Delta V$ using the following equation:

$$
V=[g]_{m \times 1} \Rightarrow \Delta V=\left[-g_{j}(x)\right]_{k \times 1} \quad \text { when } g_{j}(x)>0, j=1, \ldots, m,
$$

where $V$ is the vector of inequality constraints $(g)$, and $k$ is the degree of constraint violation $\Delta V$.

(2) Compute $\Delta_{x} g$, where $\Delta_{x} g$ are the derivatives of these constraints with respect to the solution vector ( $n$ decision variables)

$$
\Delta_{x} V=\left[\Delta_{x} g\right]_{k \times n^{\prime}} \quad x=1, \ldots, k
$$




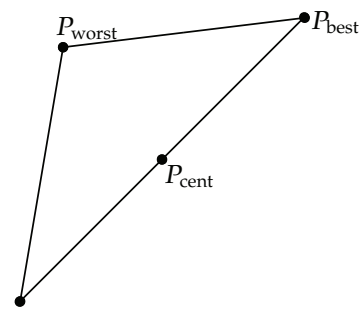

(a)

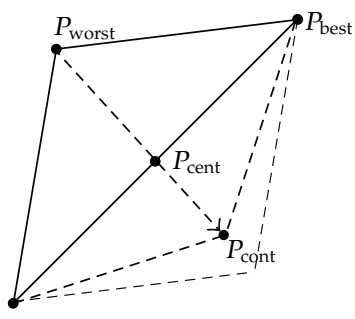

(d)

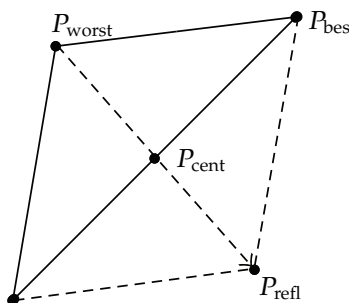

(b)

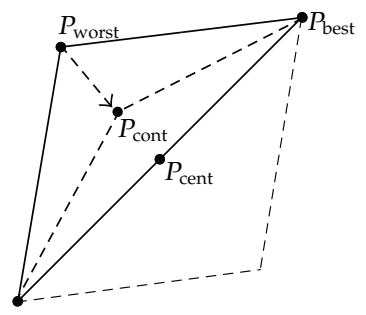

(e)

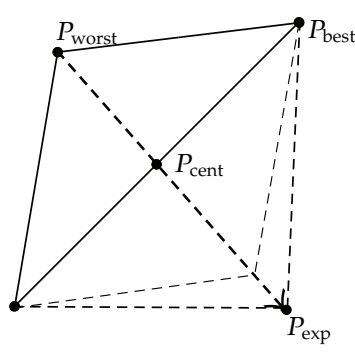

(c)

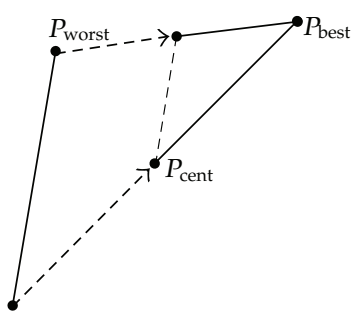

(f)

Figure 1: Illustration of the Nelder-Mead simplex method.

(3) The relationship between changes in the constraint violation $\Delta V$ and the solution vector $\Delta x$ is expressed as

$$
\Delta V=\Delta_{x} V \times \Delta x \Longrightarrow \Delta x=\Delta_{x} V^{-1} \times \Delta V
$$

(4) Compute the pseudoinverse $\Delta_{x} V^{-1}$.

(5) Update the solution vector by

$$
x^{t+1}=x^{t}+\Delta x=x^{t}+\Delta_{x} V^{-1} \times \Delta V
$$

The degree of constraint violation is adjusted according to the above procedure. In this algorithm, a "repair method" rapidly revises infeasible solutions to move them toward a feasible region. The number of constraint violations decreases and quickly vanishes with each iteration. Finally, a solution in the feasible region will be obtained.

\subsection{The Nelder-Mead Simplex Search Method}

When the search space is $n$-dimensional, the simplex consists of $n+1$ solutions [12]. As shown in Figure 1(a), in a two-dimensional search plane, a simplex is a triangle. The fitness of each solution is considered at each step of the Nelder-Mead method, and the worst solution $P_{\text {worst }}$ is identified. The centroid, $P_{\text {cent, }}$ of the remaining $n$ points is computed, and the reflection of $P_{\text {worst }}$ is determined. This reflection yields a new solution, $P_{\text {refl, }}$ which replaces $P_{\text {worst }}$, as shown in Figure $1(\mathrm{~b})$. If the solution $P_{\text {refl }}$ produced by this reflection has a higher fitness than any other solution in the simplex, the simplex is further expanded in the direction of $P_{\text {refl }}$, and $P_{\text {worst }}$ is replaced with $P_{\exp }$, as shown in Figure 1(c). 
On the other hand, if $P_{\text {refl }}$ has a comparatively low fitness, the simplex is contracted. Contraction can either be outward or inward, depending upon whether $P_{\text {refl }}$ is better or worse than $P_{\text {worst }}$, respectively. The contraction operations (i.e., $P_{\text {worst }}$ is replaced with $P_{\text {cont }}$ ) are shown in Figures 1(d) and 1(e). If neither contraction improves the worst solution in the simplex, the best point in the simplex is computed, and a shrinkage is then performed; all the points of the simplex are moved a little closer towards the best solution $\left(P_{\text {best }}\right)$, as shown in Figure 1(f).

\subsection{Particle Swarm Optimization}

In the past several years, PSO has been successfully applied in many fields [13, 14]. It has been demonstrated that the results of PSO are superior to other methods. The PSO procedure is reviewed below.

(1) Initialization. It randomly generates a swarm of potential solutions called "particles" and assigns a random velocity to each.

(2) Velocity Update. The particles are then "flown" through hyperspace by updating their own velocity. The velocity update of a particle is dynamically adjusted, subject to its own past flight and those of its companions. The velocity and position of the particles are updated by the following equations:

$$
\begin{aligned}
& V_{\mathrm{id}}^{\text {new }}(t+1)=c_{o} \times V_{\mathrm{id}}^{\text {old }}(t)+c_{1} \times \operatorname{rand}() \times\left(p_{\mathrm{id}}(t)-x_{\mathrm{id}}^{\text {old }}(t)\right)+c_{2} \times \operatorname{rand}() \times\left(p_{\mathrm{gd}}(t)-x_{\mathrm{gd}}^{\text {old }}(t)\right), \\
& x_{\mathrm{id}}^{\text {new }}(t+1)=x_{\mathrm{id}}^{\text {old }}(t)+V_{\mathrm{id}}^{\text {new }}(t+1), \\
& c_{o}=0.5+\frac{\operatorname{rand}()}{3},
\end{aligned}
$$

where $c_{1}$ and $c_{2}$ are two positive constants; $c_{0}$ is an inertia weight, and $\operatorname{rand}()$ is a random value between $(0,1)$. Zahara and Hu suggested $c_{1}=c_{2}=2$ and $c_{0}=[0.5+(\operatorname{rand}() / 2)][15]$. However, many experiments have shown that using $c_{0}=[0.5+(\operatorname{rand}() / 3)]$ provides better results. Equation (3.5) illustrates the calculation of a new velocity for each individual. The velocity of each particle is updated according to its previous velocity $\left(V_{\text {id }}\right)$, the particle's previous best location $\left(p_{\mathrm{id}}\right)$, and the global best location $\left(p_{\mathrm{gd}}\right)$. Particle velocities for each dimension are clamped to a maximum velocity $V_{\max }$. Equation (3.6) shows how each particle's position is updated in the search space.

\subsection{NM-PSO Method}

The NM-PSO optimization method [16] integrates the constraint-handling methods, the Nelder-Mead simplex search method (traditional algorithm), and the PSO algorithm (evolutionary algorithm) [17]. The PSO optimal method resists easily falling into the local best solution, but it requires many particles in an optimal process, which reduces the speed of computation. The Nelder-Mead simplex search method improves the efficiency of PSO due to its capacity for rapid convergence. However, the drawback of this method is that 
it easily falls into a local best solution. This drawback is improved by integrating the two algorithms. Combining the two algorithms and the gradient-based repair methods enables feasible optimal solutions to be found that satisfy the constraint conditions [18].

Using the advantages mentioned above, the NM-PSO method clearly overcomes the drawbacks of low convergence speed, the need for more particles, and the inability to deal with constraint conditions to accurately find optimal solutions.

\subsection{Implementation of Proposed Method}

The following section introduces the NM-PSO algorithm procedure. Assume the problem to be solved is $n$-dimensional. First produce $N(N \geqq 2 n+1)$ particles to form a swarm. For every particle that violates the constraints, use the gradient repair method to direct the infeasible solution toward the feasible region. In most cases, the repair method does move the solution to the feasible region. Arrange the results of the objective function in order of good to bad and divide the $N$ particles into $n$ particles, the $(n+1)$ th particle, and $N-(n+1)$ particles and then create three groups. First calculate the top $n$ particles and the $(n+1)$ th particle using the NM simplex method. The updated best particle is obtained and the result saved. The PSO method adjusts the $N$ particles by taking into account the position of the $(n+1)$ best particle. Through the calculation of a simple NM algorithm, the probability of finding the optimal solution was increased. This procedure for adjusting the $N$ particles involves selection of the global best particle, the selection of the neighboring best particles, and finally the velocity updates. The global best particle of the population is determined according to the sorted fitness values.

Unlike the original PSO calculation method proposed by [15], which updates the remaining particles $(N-(n+1))$, we use the PSO algorithm to update all of the $N$ particles. These two PSO algorithms combination NM methods are referred to in this paper as the original NM-PSO method and the proposed NM-PSO method, respectively. Repeat the entire NM-PSO optimization process until the condition is fulfilled. Figure 2 depicts the schematic representation of the proposed NM-PSO. Algorithm 1 shows the pseudocode of the NM-PSO algorithm embedded within the constraint-handling methods.

\section{Case Study}

The appearance and parameters of the relevant line equipment of two typical test systems are introduced. We discuss the fault current and the corresponding DOCR relationship of the coordination pairs of $\mathrm{P} / \mathrm{B}$ when a close-in three-phase short fault occurs in transmission lines.

Taking the above two test systems as examples, this study validated the feasibility of the proposed NM-PSO optimization algorithm to solve the DOCR optimal coordination problem. The results were compared with PSO and original NM-PSO algorithm. The results of the comparison demonstrate that the proposed NM-PSO algorithm is clearly better than $\mathrm{PSO}$ and original NM-PSO in terms of the objective function, the rate of convergence, and computation speed.

In this study, the multiples $2 \times n+1,5 \times n+1,10 \times n+1$, and $20 \times n+1$ were adopted as the number of populations to demonstrate the influence of the number of particles on the proposed algorithm. To observe the process and changes of convergence in the objective function, the number of iterations was set at 300 , to highlight the superior performance of the proposed system. 


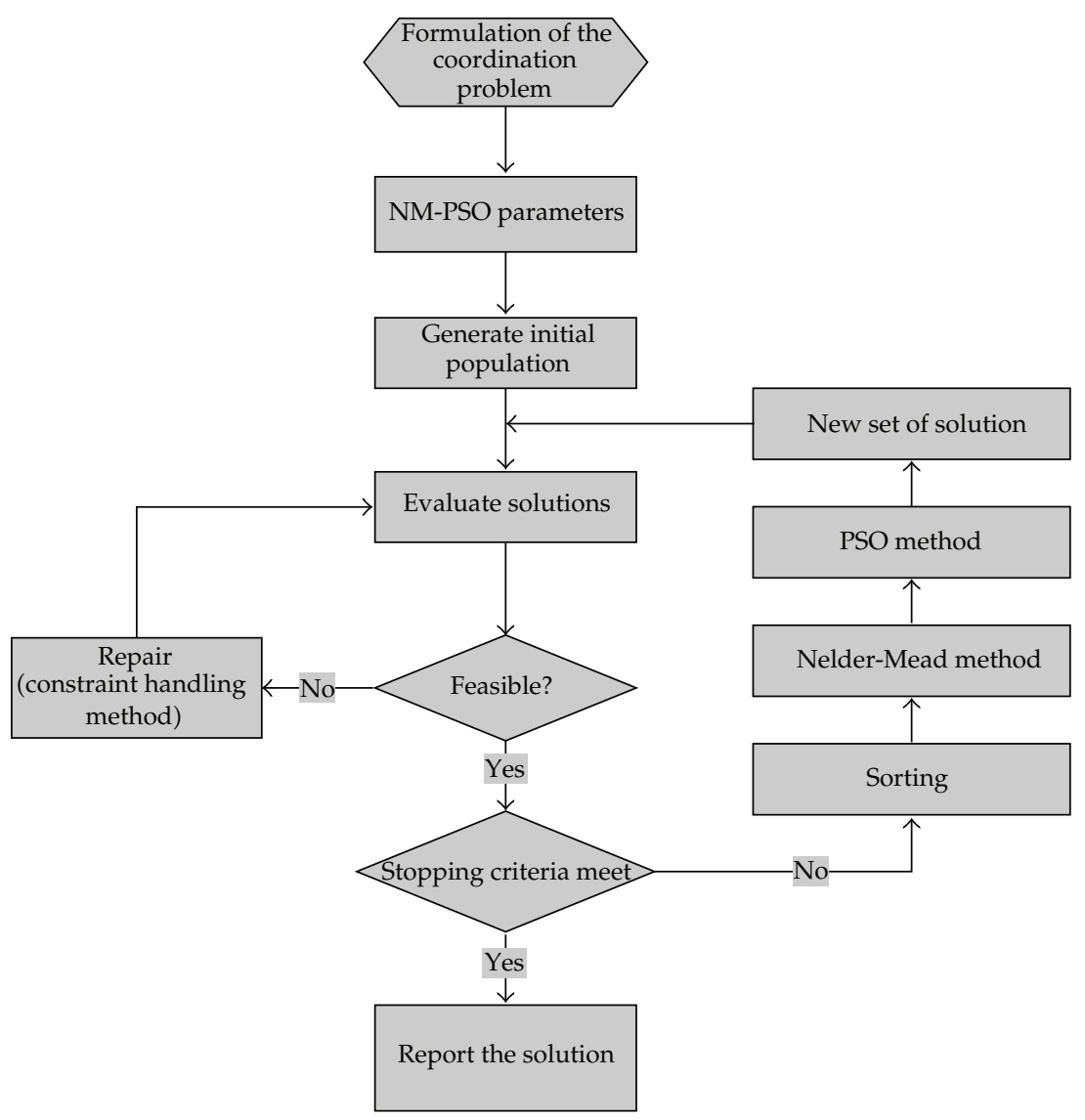

Figure 2: Flow chart of the proposed NM-PSO algorithm.

1. Initialization. Generate a population of size $N(N>(n+1))$.

Repeat

2. Constraint handling method

2.1 The Gradient Repair Method. Repair particles that violate the constraints by directing the infeasible solution toward the feasible region.

2.2 Identify solutions (that fulfill the constraint conditions) and arrange them in the order of good to bad.

3. Nelder-Mead Method. Apply NM operator to the top $n+1$ particles and update the $(n+1)$ th particle.

4. PSO Method. Apply PSO operator for updating the $N$ particles.

4.1 Selection. Select the global best particle and the neighborhood best particle from the population.

4.2 Velocity Update. Apply velocity updates to the $N$ particles until the condition is fulfilled.

Algorithm 1: Pseudocode of the proposed hybrid NM-PSO algorithm. 


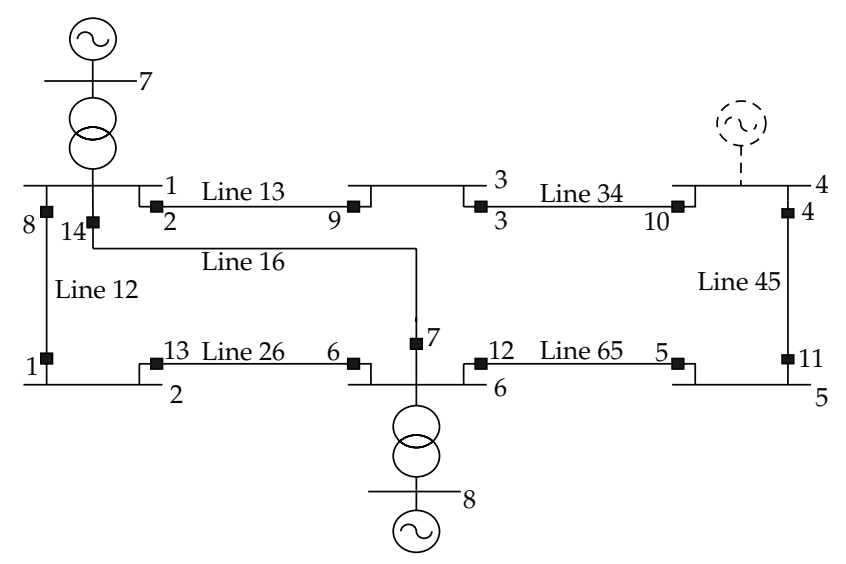

Figure 3: One-line diagram for an IEEE 8-bus test system.

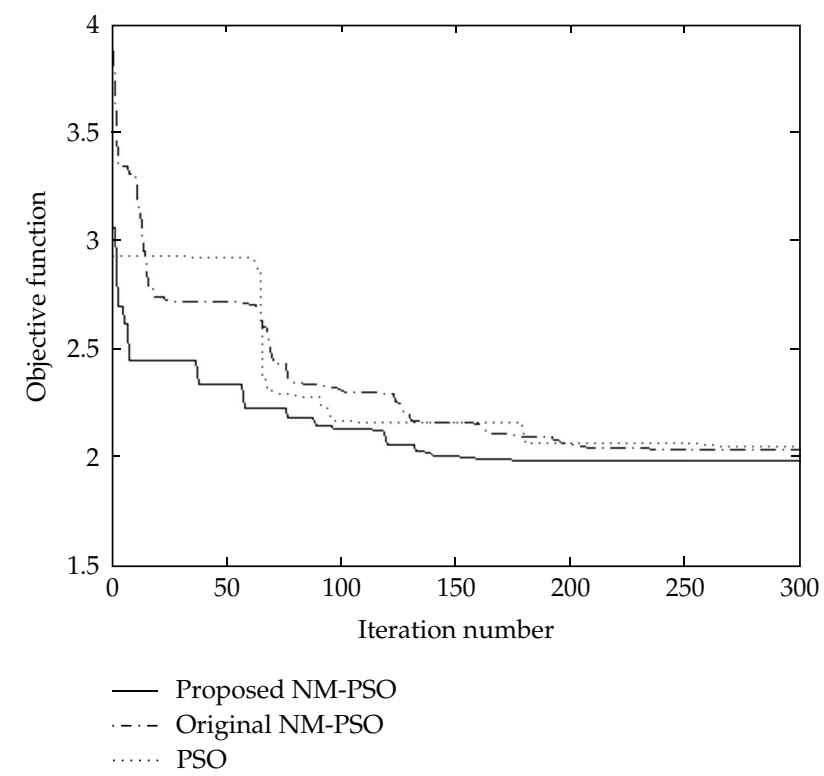

Figure 4: Convergence of PSO, original NM-PSO, and proposed NM-PSO to the optimal solution for an IEEE 8-bus test system (LP problem).

\subsection{IEEE 8-Bus Test System}

As shown in Figure 3, the 8-bus test system consists of 9 lines, 2 transformers, and 14 DOCRs. All the DOCRs have the IEEE standard inverse-time characteristics mentioned in (2.3) above. The system parameters are the same as in [6]. At bus 4, there is a link to another network modeled by a short-circuit capacity of 400 MVA. The DOCR coordination problem can be formulated as an LP problem or an MINLP problem. Additionally, there are 20 inequality constraints corresponding to each relay pair.

Table 1 illustrates the fault currents of the DOCR coordination pairs of P/B of each phase in the event of a close-in three-phase short fault of the system. If a DOCR coordination 
Table 1: P/B relays and the close-in fault currents for an IEEE 8-bus test system.

\begin{tabular}{lccc}
\hline \multirow{2}{*}{ No. } & & & Backup relay \\
\hline 1 & Current & No. & Current \\
8 & 3230 & 6 & 3230 \\
8 & 6080 & 9 & 1160 \\
2 & 6080 & 7 & 1880 \\
9 & 5910 & 1 & 993 \\
2 & 2480 & 10 & 2480 \\
3 & 5910 & 7 & 1880 \\
10 & 3550 & 2 & 3550 \\
6 & 3880 & 11 & 2340 \\
6 & 6100 & 5 & 1200 \\
13 & 6100 & 14 & 1870 \\
14 & 2980 & 8 & 2980 \\
7 & 5190 & 9 & 1160 \\
14 & 5210 & 5 & 1200 \\
7 & 5190 & 1 & 993 \\
4 & 5210 & 13 & 985 \\
11 & 3780 & 3 & 2240 \\
5 & 3700 & 12 & 3700 \\
12 & 2400 & 4 & 2400 \\
12 & 5890 & 13 & 985 \\
& 5890 & 14 & 1870 \\
\hline
\end{tabular}

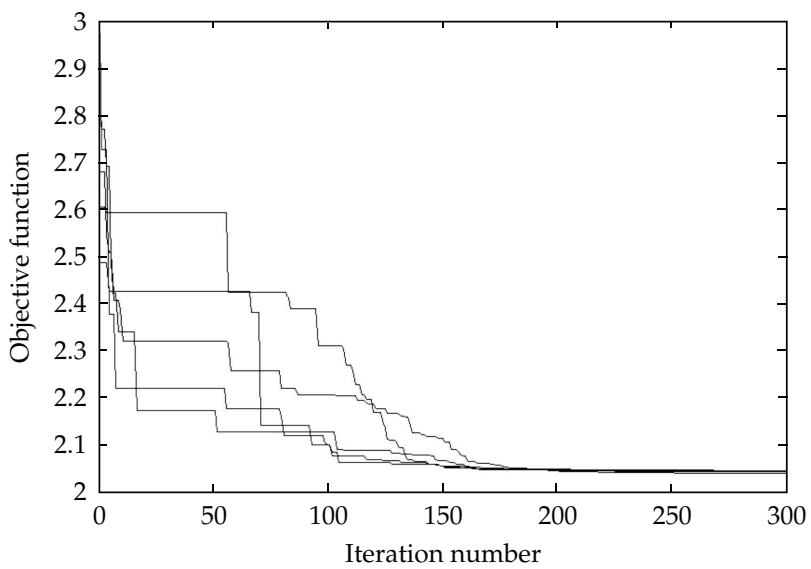

Figure 5: Convergence of the NM-PSO for five different random initial populations for an IEEE 8-bus test system (LP problem).

optimization problem with known $I_{p}$ values is assumed to be an LP problem, the results obtained using PSO, original NM-PSO, and proposed NM-PSO for the case of 300 iterations and a population size of $141(10 \times n+1$, where $n$ is the number of variables TDS of the 14 relays) are illustrated in Table 2 . The results are also compared to those of Linprog (linear programming) obtained using the MATLAB optimization toolbox.

To validate the feasibility of the proposed method, the obtained TDS values and known $I_{p}$ values were entered as constraints to obtain the results shown in Table 3 . Since 
Table 2: Optimal settings of the relays for an IEEE 8-bus test system (LP problem).

\begin{tabular}{lccccc}
\hline \multicolumn{2}{c}{ Algorithm } & Linprog & PSO & Original NM-PSO & Proposed NM-PSO \\
Relay & $I_{p}$ & TDS & TDS & TDS & TDS \\
\hline 1 & 600 & 0.1007 & 0.1000 & 0.1000 & 0.1000 \\
2 & 800 & 0.2485 & 0.2191 & 0.2260 & 0.2178 \\
3 & 500 & 0.2294 & 0.2272 & 0.2369 & 0.2240 \\
4 & 800 & 0.1115 & 0.1104 & 0.1234 & 0.1097 \\
5 & 600 & 0.1003 & 0.1000 & 0.1075 & 0.1000 \\
6 & 500 & 0.3858 & 0.3844 & 0.3882 & 0.3850 \\
7 & 600 & 0.1103 & 0.1030 & 0.1233 & 0.1028 \\
8 & 500 & 0.3575 & 0.3645 & 0.3587 & 0.3537 \\
9 & 600 & 0.1001 & 0.1000 & 0.1007 & 0.1000 \\
10 & 500 & 0.2947 & 0.3476 & 0.2963 & 0.2943 \\
11 & 600 & 0.1794 & 0.2102 & 0.1958 & 0.1786 \\
12 & 500 & 0.5591 & 0.6000 & 0.6194 & 0.5954 \\
13 & 600 & 0.1007 & 0.1000 & 0.1000 & 0.1000 \\
14 & 800 & 0.1094 & 0.1000 & 0.1030 & 0.1000 \\
\hline \multicolumn{7}{l}{ Obj-Fun } & 1.9640 & 2.0468 & 2.0278 & 1.9783 \\
\hline
\end{tabular}

Table 3: Operating time of P/B relays for an IEEE 8-bus test system (LP problem).

\begin{tabular}{|c|c|c|c|c|}
\hline \multicolumn{5}{|c|}{ Proposed NM-PSO method } \\
\hline \multicolumn{2}{|c|}{ Backup relay } & \multicolumn{2}{|c|}{ Primary relay } & \multirow{2}{*}{ Constraint value } \\
\hline No. & Operating time & No. & Operating time & \\
\hline 6 & 0.3134 & 1 & 0.1130 & 0.2004 \\
\hline 9 & 1.0422 & 8 & 0.1110 & 0.9312 \\
\hline 7 & 0.3413 & 8 & 0.1110 & 0.2303 \\
\hline 1 & 1.6338 & 2 & 0.1412 & 1.4926 \\
\hline 10 & 0.3875 & 9 & 0.1875 & 0.2000 \\
\hline 7 & 0.3413 & 2 & 0.1412 & 0.2001 \\
\hline 2 & 0.3551 & 3 & 0.1551 & 0.2000 \\
\hline 11 & 0.3762 & 10 & 0.1760 & 0.2002 \\
\hline 5 & 0.9522 & 6 & 0.1203 & 0.8319 \\
\hline 14 & 0.6439 & 6 & 0.1203 & 0.5236 \\
\hline 8 & 0.3320 & 13 & 0.1313 & 0.2007 \\
\hline 9 & 1.0422 & 14 & 0.0808 & 0.9614 \\
\hline 5 & 0.9522 & 7 & 0.0515 & 0.9007 \\
\hline 1 & 1.6338 & 14 & 0.0808 & 1.5530 \\
\hline 13 & 1.6758 & 7 & 0.0515 & 1.6243 \\
\hline 3 & 0.3585 & 4 & 0.1584 & 0.2001 \\
\hline 12 & 0.3848 & 11 & 0.1578 & 0.2270 \\
\hline 4 & 0.4000 & 5 & 0.2000 & 0.2000 \\
\hline 13 & 1.6758 & 12 & 0.1943 & 1.4815 \\
\hline 14 & 0.6439 & 12 & 0.1943 & 0.4496 \\
\hline
\end{tabular}




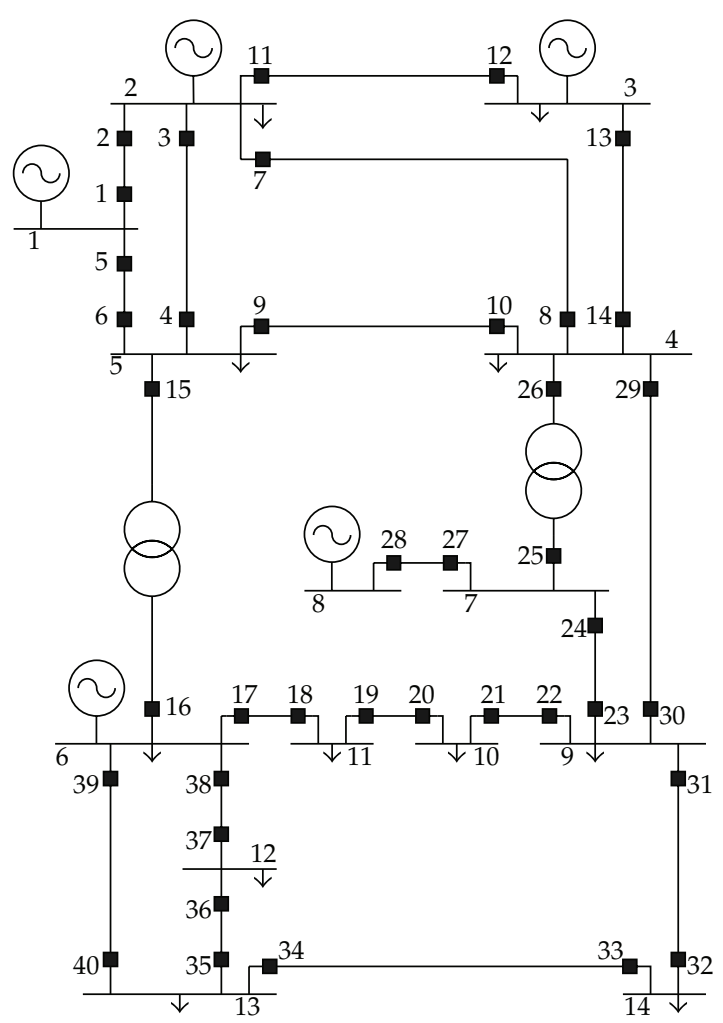

Figure 6: One-line diagram for an IEEE 14-bus test system.

the proposed method satisfies all constraints (i.e., CTI $\geqq 0.2$ ), the best coordination setting for DOCR can be efficiently completed.

As expected, the proposed NM-PSO yields better objective function results than PSO and original NM-PSO. Figure 4 shows that proposed NM-PSO nearly reached the global optimum after 189 iterations. The results of this proposed NM-PSO algorithm reveal better convergence.

To analyze the convergence consistency of the proposed NM-PSO algorithm when solving the LP problem in the case of different initial values, this study randomly performed the proposed method five times. As seen in Figure 5, the proposed NM-PSO algorithm can reduce the objective function to the same value after nearly 200 iterations. The convergence of the proposed NM-PSO is evidently not affected by different initial values.

\subsection{IEEE 14-Bus Test System}

The IEEE 14-bus test system consists of 5 generators, 2 power transformers, 20 transmission lines, and 40 DOCRs, as shown in Figure 6. The system parameters are given in [19]. The voltage level and the power base of this system are $138 \mathrm{kV}$ and $100 \mathrm{MVA}$, respectively.

It is assumed that the DOCRs all have the standard IEEE inverse-time characteristics as an IEEE 8-bus test system. Table 4 reveals the $\mathrm{P} / \mathrm{B}$ relay pairs and the corresponding fault 
Table 4: $\mathrm{P} / \mathrm{B}$ relays and the close-in fault currents for an IEEE 14-bus test system.

\begin{tabular}{|c|c|c|c|c|c|c|c|c|c|c|c|}
\hline \multicolumn{2}{|c|}{ Primary relay } & \multicolumn{2}{|c|}{ Backup relay } & \multicolumn{2}{|c|}{ Primary relay } & \multicolumn{2}{|c|}{ Backup relay } & \multicolumn{2}{|c|}{ Primary relay } & \multicolumn{2}{|c|}{ Backup relay } \\
\hline No. & Current & No. & Current & No. & Current & No. & Current & No. & Current & No. & Current \\
\hline 1 & 11650 & 6 & 654 & 8 & 3880 & 30 & 188 & 19 & 955 & 17 & 955 \\
\hline 5 & 12400 & 2 & 1980 & 29 & 4720 & 7 & 1220 & 18 & 725 & 20 & 725 \\
\hline 2 & 4260 & 4 & 750 & 29 & 4720 & 9 & 1990 & 22 & 1930 & 29 & 499 \\
\hline 2 & 4260 & 12 & 875 & 29 & 4720 & 13 & 1070 & 22 & 1930 & 24 & 1160 \\
\hline 2 & 4260 & 8 & 723 & 29 & 4720 & 25 & 449 & 22 & 1930 & 32 & 280 \\
\hline 3 & 7310 & 1 & 3920 & 6 & 3830 & 3 & 1280 & 23 & 1200 & 21 & 434 \\
\hline 3 & 7310 & 12 & 848 & 6 & 3830 & 10 & 1990 & 23 & 1200 & 29 & 499 \\
\hline 3 & 7310 & 8 & 689 & 6 & 3830 & 16 & 560 & 23 & 1200 & 32 & 281 \\
\hline 11 & 7180 & 4 & 725 & 4 & 3920 & 5 & 1370 & 30 & 1810 & 21 & 424 \\
\hline 11 & 7180 & 1 & 3920 & 4 & 3920 & 16 & 562 & 30 & 1810 & 24 & 1130 \\
\hline 11 & 7180 & 8 & 695 & 4 & 3920 & 10 & 1990 & 30 & 1810 & 32 & 275 \\
\hline 7 & 7330 & 1 & 3920 & 15 & 4610 & 5 & 1360 & 31 & 2060 & 21 & 428 \\
\hline 7 & 7330 & 4 & 716 & 15 & 4610 & 3 & 1280 & 31 & 2060 & 24 & 1150 \\
\hline 7 & 7330 & 12 & 845 & 15 & 4610 & 10 & 1970 & 31 & 2060 & 29 & 494 \\
\hline 13 & 3280 & 11 & 1380 & 9 & 3260 & 5 & 1390 & 27 & 2030 & 26 & 1230 \\
\hline 12 & 3130 & 14 & 1250 & 9 & 3260 & 3 & 1310 & 27 & 2030 & 23 & 808 \\
\hline 26 & 4640 & 9 & 2080 & 9 & 3260 & 16 & 569 & 25 & 1430 & 28 & 633 \\
\hline 26 & 4640 & 13 & 1120 & 16 & 1490 & 40 & 201 & 25 & 1430 & 23 & 806 \\
\hline 26 & 4640 & 7 & 1270 & 16 & 1490 & 18 & 388 & 24 & 1870 & 26 & 1230 \\
\hline 26 & 4640 & 30 & 179 & 16 & 1490 & 37 & 51 & 24 & 1870 & 28 & 634 \\
\hline 10 & 3110 & 13 & 1140 & 17 & 2210 & 15 & 1110 & 37 & 572 & 35 & 572 \\
\hline 10 & 3110 & 7 & 1290 & 17 & 2210 & 37 & 51 & 36 & 781 & 38 & 781 \\
\hline 10 & 3110 & 25 & 495 & 17 & 2210 & 40 & 199 & 35 & 1480 & 33 & 368 \\
\hline 10 & 3110 & 30 & 190 & 39 & 2400 & 15 & 1120 & 35 & 1480 & 39 & 1110 \\
\hline 14 & 4030 & 9 & 2090 & 39 & 2400 & 18 & 389 & 34 & 1390 & 36 & 284 \\
\hline 14 & 4030 & 7 & 1270 & 39 & 2400 & 37 & 47 & 34 & 1390 & 39 & 1110 \\
\hline 14 & 4030 & 25 & 489 & 38 & 2530 & 15 & 1110 & 40 & 654 & 36 & 285 \\
\hline 14 & 4030 & 30 & 188 & 38 & 2530 & 40 & 191 & 40 & 654 & 33 & 370 \\
\hline 8 & 3880 & 9 & 2090 & 38 & 2530 & 18 & 386 & 32 & 547 & 34 & 547 \\
\hline 8 & 3880 & 25 & 489 & 21 & 564 & 19 & 564 & 33 & 783 & 31 & 783 \\
\hline 8 & 3880 & 13 & 1120 & 20 & 1310 & 22 & 1310 & $x$ & $\mathrm{x}$ & $\mathrm{x}$ & $\mathrm{x}$ \\
\hline
\end{tabular}

currents passing through them for a close-in fault in this network. There are 92 inequality constraints corresponding to each relay pair.

This study also used PSO, original NM-PSO, and proposed NM-PSO to solve the DOCR coordination optimization of the MINLP problem, which required obtaining the TDS and $I_{p}$ values of each DOCR. The results after 300 iterations with a population size of 1601 $\left(20 \times n+1\right.$, where $n$ is the number of TDS and $I_{p}$ variables of the 40 relays) are shown in Table 5 . The integer $I_{p}$ can be directly applied in the current intelligent electronic device (IED) settings.

Figure 7 shows the results of the comparison demonstrate that the proposed NM-PSO algorithm is clearly better than PSO and original NM-PSO in terms of the objective function, the rate of convergence, and computation speed. 
Table 5: Optimal settings of the relays for an IEEE 14-bus test system (MINLP problem).

\begin{tabular}{|c|c|c|c|c|c|c|}
\hline \multirow{2}{*}{$\begin{array}{l}\text { Algorithm } \\
\text { relay }\end{array}$} & \multicolumn{2}{|c|}{ PSO } & \multicolumn{2}{|c|}{ Original NM-PSO } & \multicolumn{2}{|c|}{ Proposed NM-PSO } \\
\hline & $I_{p}$ & TDS & $I_{p}$ & TDS & $I_{p}$ & TDS \\
\hline 1 & 881 & 0.1746 & 807 & 0.2024 & 736 & 0.3038 \\
\hline 2 & 579 & 0.1000 & 515 & 0.1729 & 540 & 0.1211 \\
\hline 3 & 408 & 0.2746 & 422 & 0.2108 & 437 & 0.1418 \\
\hline 4 & 216 & 0.1239 & 261 & 0.1574 & 237 & 0.1184 \\
\hline 5 & 758 & 0.1059 & 700 & 0.1860 & 700 & 0.1184 \\
\hline 6 & 296 & 0.1607 & 227 & 0.2694 & 200 & 0.1020 \\
\hline 7 & 532 & 0.2192 & 435 & 0.1187 & 419 & 0.1718 \\
\hline 8 & 226 & 0.1335 & 206 & 0.1605 & 270 & 0.1452 \\
\hline 9 & 444 & 0.1812 & 504 & 0.1454 & 537 & 0.1258 \\
\hline 10 & 488 & 0.3347 & 528 & 0.1154 & 450 & 0.1523 \\
\hline 11 & 477 & 0.1717 & 516 & 0.2881 & 550 & 0.1006 \\
\hline 12 & 352 & 0.1015 & 295 & 0.2357 & 220 & 0.1414 \\
\hline 13 & 394 & 0.1546 & 355 & 0.2212 & 350 & 0.1307 \\
\hline 14 & 418 & 0.1000 & 362 & 0.2003 & 358 & 0.1250 \\
\hline 15 & 333 & 0.1342 & 316 & 0.2404 & 359 & 0.1872 \\
\hline 16 & 149 & 0.1451 & 126 & 0.2268 & 117 & 0.2302 \\
\hline 17 & 216 & 0.3880 & 268 & 0.2414 & 177 & 0.3258 \\
\hline 18 & 110 & 0.1172 & 101 & 0.1418 & 100 & 0.1301 \\
\hline 19 & 164 & 0.2737 & 168 & 0.1379 & 108 & 0.3402 \\
\hline 20 & 151 & 0.2322 & 170 & 0.1708 & 211 & 0.1057 \\
\hline 21 & 100 & 0.3998 & 121 & 0.1308 & 115 & 0.1534 \\
\hline 22 & 174 & 0.6243 & 339 & 0.1427 & 326 & 0.1473 \\
\hline 23 & 119 & 0.5556 & 193 & 0.1457 & 150 & 0.2554 \\
\hline 24 & 147 & 0.7269 & 307 & 0.1543 & 315 & 0.1437 \\
\hline 25 & 133 & 0.2076 & 127 & 0.1483 & 102 & 0.3358 \\
\hline 26 & 260 & 0.3107 & 351 & 0.1631 & 399 & 0.2024 \\
\hline 27 & 150 & 0.1005 & 150 & 0.2364 & 150 & 0.1641 \\
\hline 28 & 204 & 0.1230 & 127 & 0.3070 & 154 & 0.1779 \\
\hline 29 & 245 & 0.2288 & 200 & 0.2564 & 200 & 0.2033 \\
\hline 30 & 150 & 0.1000 & 100 & 0.2557 & 100 & 0.2124 \\
\hline 31 & 241 & 0.1776 & 247 & 0.1765 & 164 & 0.2053 \\
\hline 32 & 100 & 0.1066 & 100 & 0.1000 & 100 & 0.1001 \\
\hline 33 & 177 & 0.1000 & 108 & 0.2252 & 100 & 0.1511 \\
\hline 34 & 123 & 0.2518 & 100 & 0.2858 & 112 & 0.2285 \\
\hline 35 & 100 & 0.3861 & 100 & 0.2349 & 100 & 0.3681 \\
\hline 36 & 146 & 0.1132 & 121 & 0.1311 & 100 & 0.1300 \\
\hline 37 & 36 & 0.1000 & 10 & 0.2869 & 11 & 0.3143 \\
\hline 38 & 238 & 0.2182 & 245 & 0.1527 & 249 & 0.1892 \\
\hline 39 & 268 & 0.2090 & 269 & 0.1850 & 266 & 0.1596 \\
\hline 40 & 113 & 0.1000 & 100 & 0.2529 & 100 & 0.1485 \\
\hline Obj-Fun & \multicolumn{2}{|c|}{4.2233} & \multicolumn{2}{|c|}{3.6362} & \multicolumn{2}{|c|}{3.1829} \\
\hline
\end{tabular}




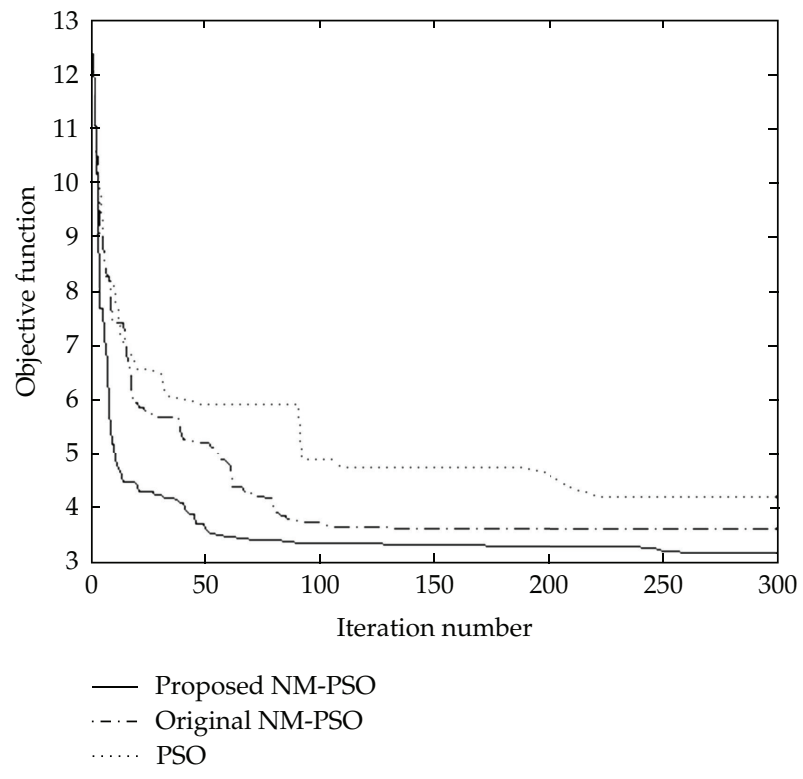

Figure 7: Convergence of PSO, original NM-PSO, and proposed NM-PSO to the optimal solution for an IEEE 14-bus test system (MINLP problem).

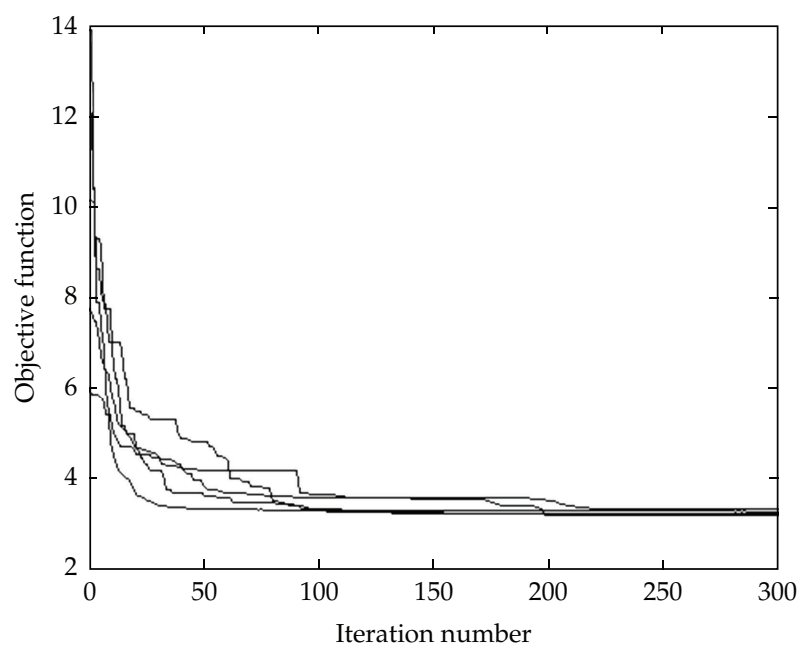

Figure 8: Convergence of the proposed NM-PSO for five different random initial populations for an IEEE 14-bus test system (MINLP problem).

To analyze the consistency in convergence of the proposed NM-PSO when solving MINLP problems, this study randomly executed the proposed method five times. As seen from Figure 8, the proposed NM-PSO can reduce the objective function to almost the same value after approximately 220 iterations. Results show that the convergence of the proposed NM-PSO is not seriously influenced by optimization problems of high complexity.

In addition to convergence rate, we investigated the final convergence values of the objective function (Obj-Fun). Figures 9(a) and 9(b) show the results of LP coordination 


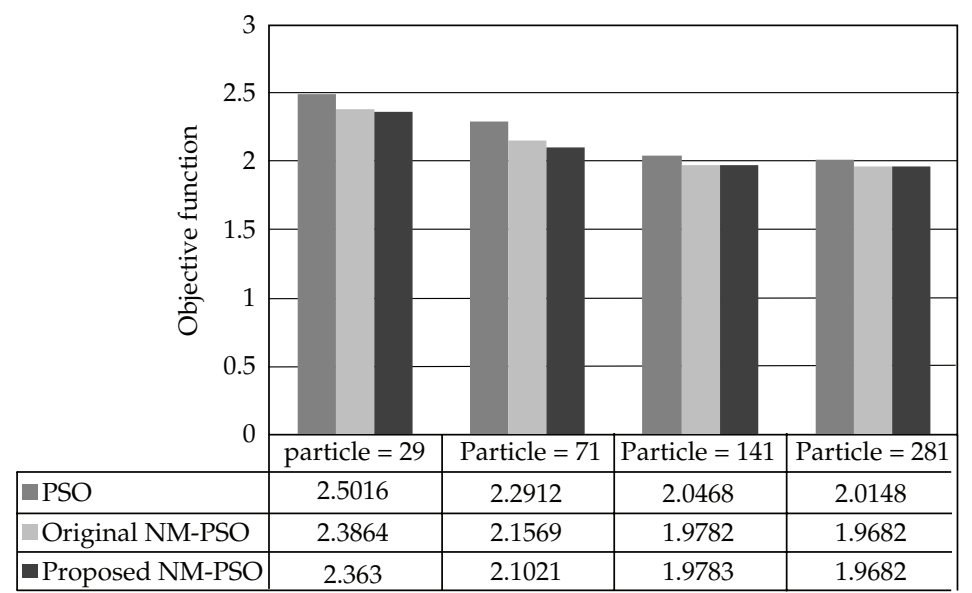

(a) IEEE 8-bus test system (LP problem)

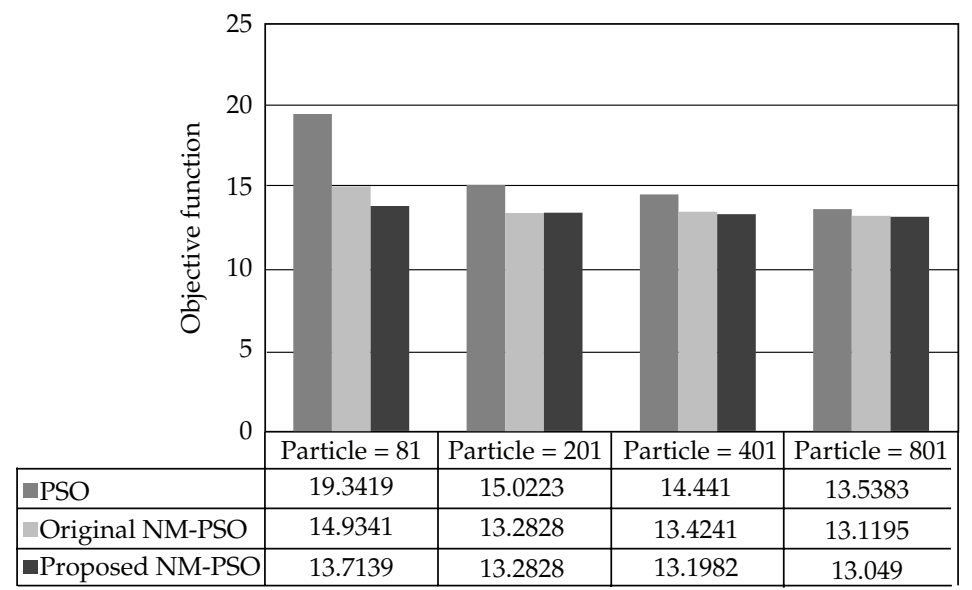

(b) IEEE 14-bus test system (LP problem)

Figure 9: Objective function values by PSO, original NM-PSO, and proposed NM-PSO algorithms for the LP problem with $n=40$ for four different populations.

problems, for the same number of iterations (300) and for four different populations $2 \times n+$ $1,5 \times n+1,10 \times n+1$, and $20 \times n+1$.

It can clearly be seen that the proposed NM-PSO method results in better Obj-Fun values than the PSO and original NM-PSO algorithm. For the more complicated MINLP problem shown in Figures 10(a) and 10(b), the proposed NM-PSO after 300 iterations results in even better Obj-Fun values in the case of particles $2 \times n+1$ than the Obj-Fun values of PSO algorithm in the case of particles $20 \times n+1$. Hence, the proposed method produces better results than the PSO algorithm using fewer particles (less computation time).

\section{Conclusions}

In this paper, the DOCR coordination problem is formulated as a constrained optimization problem. It can be concluded that the proposed NM-PSO optimization algorithm is applicable 


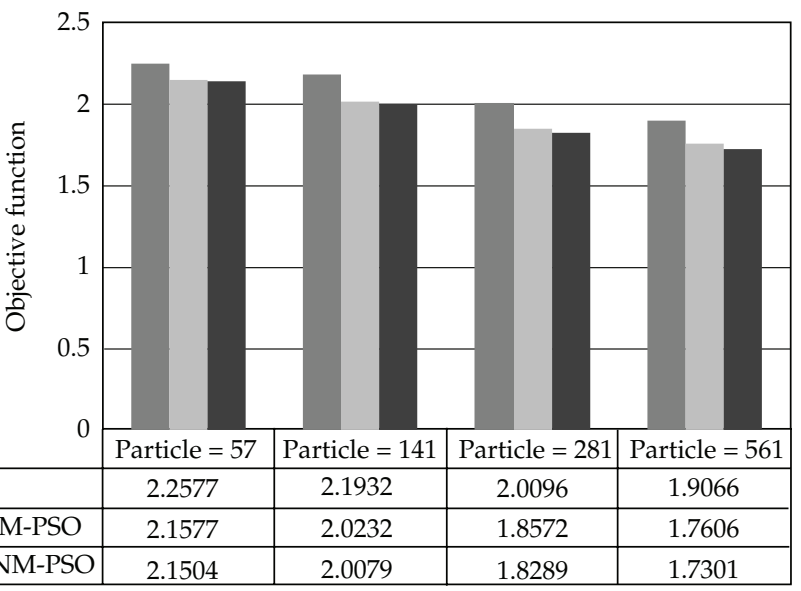

(a) IEEE 8-bus test system (MINLP problem)

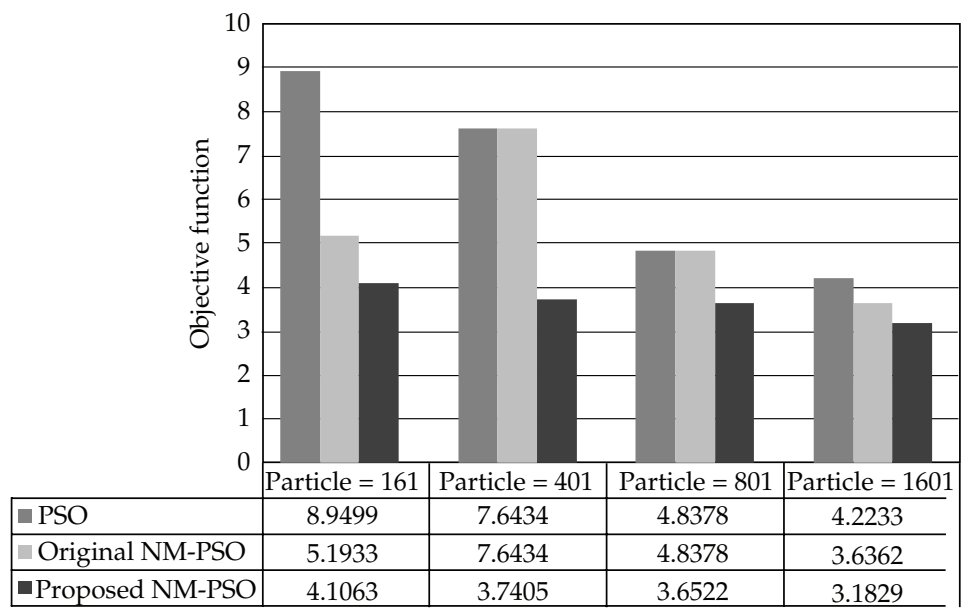

(b) IEEE 14-bus test system (MINLP problem)

Figure 10: Objective function values by PSO, original NM-PSO, and proposed NM-PSO algorithms for the MINLP problem with $n=80$ for four different populations.

to the DOCR coordination optimization of a distribution system. In contrast to other methods in the literature that only find TDS, the algorithm proposed in this study obtains $I_{p}$ and TDS values simultaneously, and the $I_{p}$ values can be represented by integers for applications in the IED setting to complete a more comprehensive coordination optimization. The proposed method makes use of the advantages of both the NM and PSO methods, while overcoming the drawbacks associated with these methods. Regardless of whether LP or MINLP is used to solve a coordination optimization problem, we have demonstrated that the proposed algorithm performs better than PSO and original NM-PSO algorithm in terms of computation speed, rate of convergence, and objective function value. The reduction in the DOCR operating time in our results demonstrates that the proposed method can be adopted for determining the optimum settings of DOCRs. 


\section{References}

[1] A. J. Urdaneta, R. Nadira, and L. G. Perez Jimenez, "Optimal coordination of directional overcurrent relays in interconnected power systems," IEEE Transactions on Power Delivery, vol. 3, pp. 903-911, 1988.

[2] H. A. Abyaneh, M. Al-Dabbagh, H. K. Karegar, S. H. H. Sadeghi, and R. A. J. Khan, "A new optimal approach for coordination of overcurrent relays in interconnected power systems," IEEE Transactions on Power Delivery, vol. 18, no. 2, pp. 430-435, 2003.

[3] D. Birla, R. P. Maheshwari, and H. O. Gupta, "A new nonlinear directional overcurrent relay coordination technique, and banes and boons of near-end faults based approach," IEEE Transactions on Power Delivery, vol. 21, no. 3, pp. 1176-1182, 2006.

[4] A. S. Noghabi, J. Sadeh, and H. R. Mashhadi, "Considering different network topologies in optimal overcurrent relay coordination using a hybrid GA," IEEE Transactions on Power Delivery, vol. 24, no. 4, pp. 1857-1863, 2009.

[5] A. S. Noghabi, H. R. Mashhadi, and J. Sadeh, "Optimal coordination of directional overcurrent relays considering different network topologies using interval linear programming," IEEE Transactions on Power Delivery, vol. 25, no. 3, pp. 1348-1354, 2010.

[6] P. P. Bedekar and S. R. Bhide, "Optimum coordination of directional overcurrent relays using the hybrid GA-NLP approach," IEEE Transactions on Power Delivery, vol. 26, no. 1, pp. 109-119, 2011.

[7] H. H. Zeineldin, E. F. El-Saadany, and M. M. A. Salama, "Optimal coordination of overcurrent relays using a modified particle swarm optimization," Electric Power Systems Research, vol. 76, no. 11, pp. 988-995, 2006.

[8] M. M. Mansour, S. F. Mekhamer, and N. E. S. El-Kharbawe, “A modified particle swarm optimizer for the coordination of directional overcurrent relays," IEEE Transactions on Power Delivery, vol. 22, no. 3, pp. 1400-1410, 2007.

[9] R. C. Eberhart and J. Kennedy, "A new optimizer using particle swarm theory," in Proceedings of the 6th International Symposium on Micro Machine and Human Science, pp. 39-43, Nagoya, Japan, October 1995.

[10] P. Chootinan and A. Chen, "Constraint handling in genetic algorithms using a gradient-based repair method," Computers and Operations Research, vol. 33, no. 8, pp. 2263-2281, 2006.

[11] Y. Dong, J. F. Tang, B. D. Xu, and D. Wang, “An application of swarm optimization to nonlinear programming," Computers and Mathematics with Applications, vol. 49, no. 11-12, pp. 1655-1668, 2005.

[12] J. H. Mathews, Numerical Methods Using Matlab, Prentice-Hall, Upper Saddle River, NJ, USA, 2004.

[13] T. Niknam, H. D. Mojarrad, and M. Nayeripour, "A new hybrid fuzzy adaptive particle swarm optimization for non-convex economic dispatch," International Journal of Innovative Computing, Information and Control, vol. 7, no. 1, pp. 189-202, 2011.

[14] C. H. Lin, J. L. Chen, and Z. L. Gaing, "Combining biometric fractal pattern and particle swarm optimization-based classifier for fingerprint recognition," Mathematical Problems in Engineering, vol. 2010, Article ID 328676, 14 pages, 2010.

[15] E. Zahara and C. H. Hu, "Solving constrained optimization problems with hybrid particle swarm optimization," Engineering Optimization, vol. 40, no. 11, pp. 1031-1049, 2008.

[16] E. Zahara and A. Liu, "Solving parameter identification problem by hybrid particle swarm optimization," in Proceedings of the International MultiConference of Engineers and Computer Scientists 2010 (IMECS '10), pp. 36-38, March 2010.

[17] A. Liu and E. Zahara, "Parameter identification problem using particle swarm optimization," in Proceedings of the 5th International Conference on Natural Computation (ICNC '09), pp. 275-278, Tianjin, China, August 2009.

[18] Y. T. Kao, E. Zahara, and I. W. Kao, "A hybridized approach to data clustering," Expert Systems with Applications, vol. 34, no. 3, pp. 1754-1762, 2008.

[19] S. Kamel and M. Kodsi, "Modeling and simulation of IEEE 14 bus system with facts controllers," Tech. Rep., 2009. 


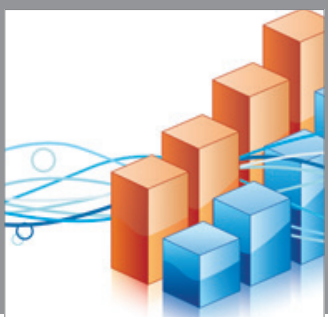

Advances in

Operations Research

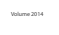

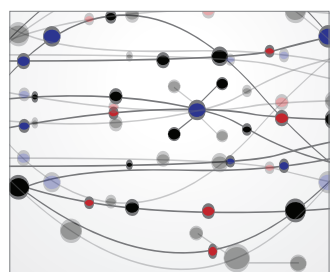

\section{The Scientific} World Journal
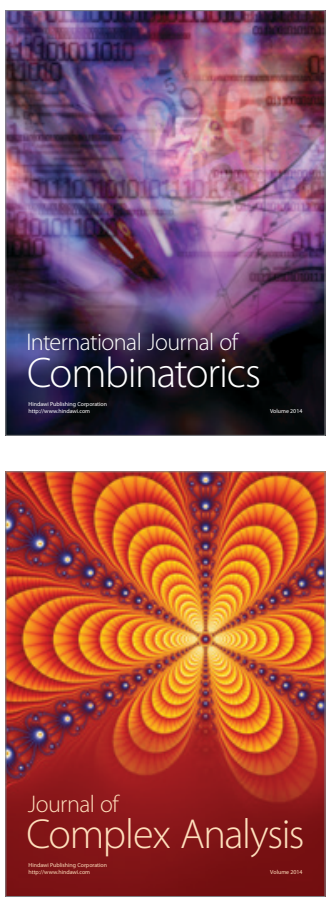

International Journal of

Mathematics and

Mathematical

Sciences
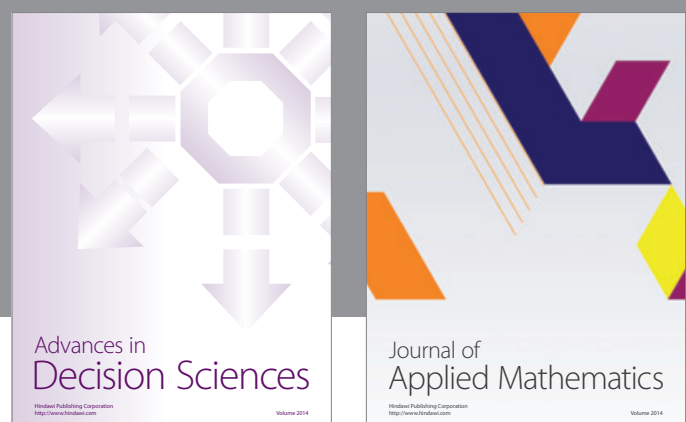

Journal of

Applied Mathematics
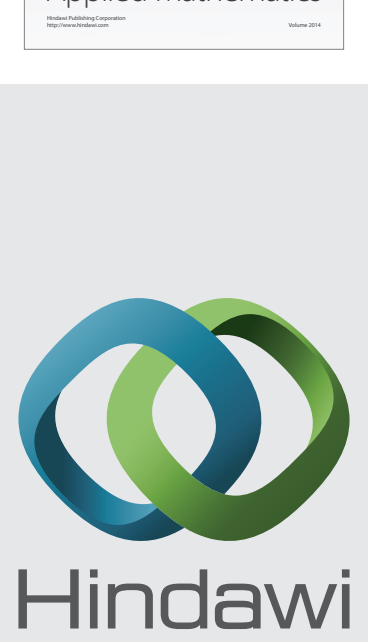

Submit your manuscripts at http://www.hindawi.com
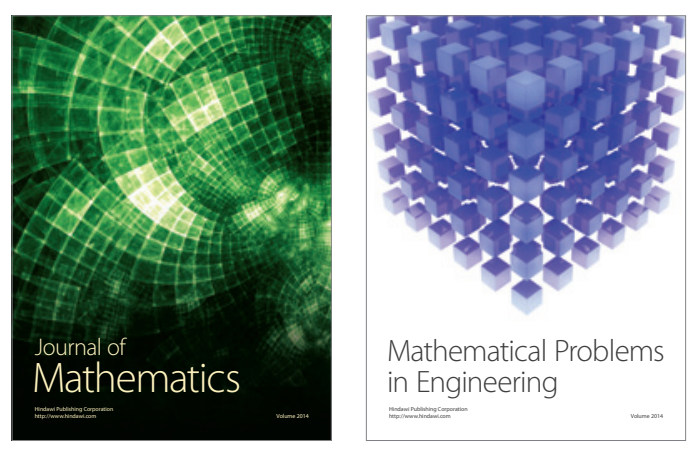

Mathematical Problems in Engineering
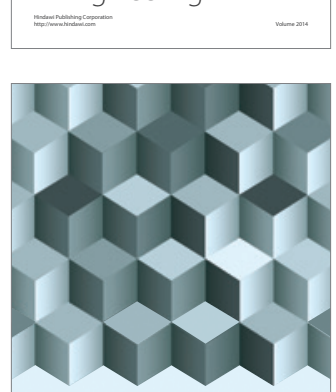

Journal of

Function Spaces
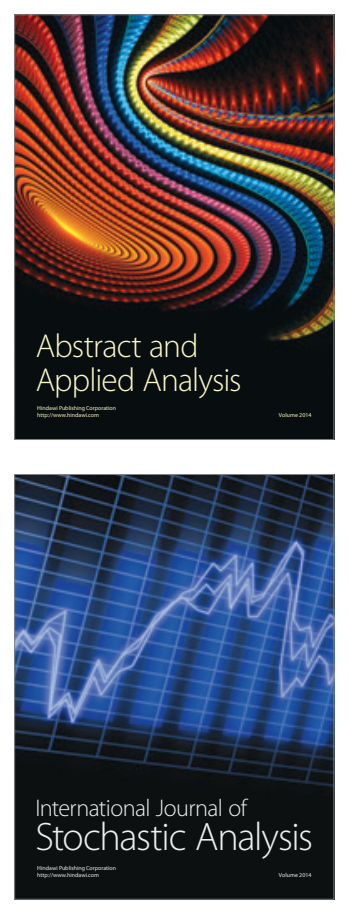

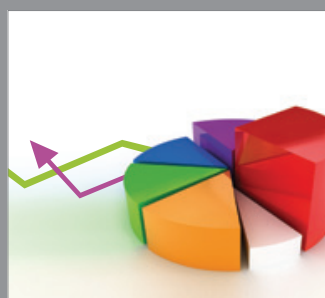

ournal of

Probability and Statistics

Promensencen
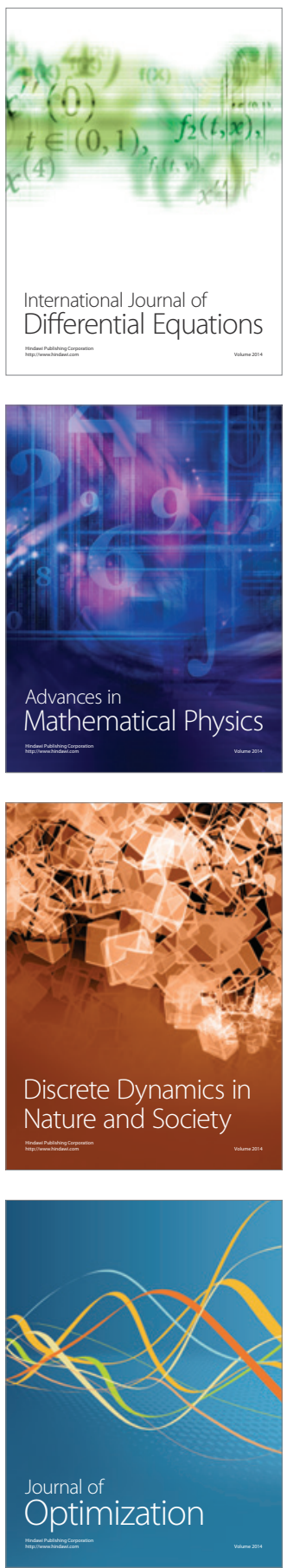